\title{
Correlates of protective immunity for Ebola vaccines: implications for regulatory approval by the animal rule
}

Nancy J. Sullivan*, Julie E. Martin ${ }^{\ddagger}$ Barney S. Graham ${ }^{\ddagger}$ and Gary J. Nabel§

Abstract | Ebola virus infection is a highly lethal disease for which there are no effective therapeutic or preventive treatments. Several vaccines have provided immune protection in laboratory animals, but because outbreaks occur unpredictably and sporadically, vaccine efficacy cannot be proven in human trials, which is required for traditional regulatory approval. The Food and Drug Administration has introduced the 'animal rule', to allow laboratory animal data to be used to show efficacy when human trials are not logistically feasible. In this Review, we describe immune correlates of vaccine protection against Ebola virus in animals. This research provides a basis for bridging the gap from basic research to human vaccine responses in support of the licensing of vaccines through the animal rule.

The filoviruses, Ebola virus and Marburg virus, cause outbreaks of highly lethal haemorrhagic fever. Filovirus mortality rates average greater than $50 \%$, and the highest recorded mortality rates have been for Ebola Zaire virus (88\%) and Marburg Angola virus (90\%) ${ }^{1}$. After infection, these haemorrhagic fever viruses induce a sustained high fever followed by aberrant coagulation and vascular permeability, which causes bleeding, bruising and a rash. After the asymptomatic incubation period, which can last days to weeks, symptoms of a typical viral infection emerge; headache, nausea, fever and malaise precede more serious haemorrhagic symptoms and, in fatal cases, death results from multi-organ failure owing to shock. The treatments for filovirus infection are palliative, and consist primarily of supportive care, including hydration and pain management.

Most filovirus outbreaks have occurred in equatorial Section, ${ }^{\ddagger}$ Clinical Trials Core Laboratory and Viral Pathogenesis Laboratory, \$Virology Laboratory, Vaccine Research Center, National Institute for Allergy and Infectious Disease, National Institutes of Health, 40 Convent Drive, MSC 3005 , Bethesda, Maryland 20892, USA.

Correspondence to N.J.S. e-mail:nsullivan@nih.gov doi:10.1038/nrmicro2129

Africa, ranging from the Ivory Coast in the west, to Uganda in the east, occasional Marburg virus in Kenya and as far south as Angola, but are concentrated in Central Africa, particularly the Democratic Republic of the Congo, the Republic of the Congo and Uganda. In most outbreaks, epidemiological investigation has linked the index human case to animal contact, either non-human primate or antelope, or travel into mines and presumed exposure to bats ${ }^{1}$. Bats have recently been identified as a possible natural reservoir for filoviruses after the recovery of three Marburg virus isolates from bats ${ }^{2}$. Transmission between humans generally requires close contact with blood or body fluids during care for the ill and burial practices that include bathing the deceased. Outbreak control is facilitated by patient isolation, the use of protective equipment and avoidance of contaminated needles. Public health responses are directed toward surveillance, diagnosis and community education.

There is no effective treatment or cure; therefore, vaccine development is crucially important as a strategy for minimizing the public health, societal and economic impacts of natural filovirus outbreaks. However, vaccine efficacy testing for Ebola virus is difficult: there is no readily identifiable high-risk population that can be targeted for a placebo-controlled clinical efficacy trial because outbreaks are unpredictable and sporadic, both geographically and temporally.

\section{Regulatory approval of vaccines}

The pathway to a vaccine licence includes an assessment of toxicity, immune response and efficacy in pre-clinical animal studies, clinical safety studies (Phase I and II) and clinical efficacy studies (Phase III) (TABLE 1). Clinical efficacy studies usually evaluate incidence rates in vaccinated individuals compared with placebo recipients, and are typically performed in populations that are known to be at risk for a particular condition and in a setting where the known incidence has been defined, to allow study design and assess feasibility. 
Table 1 | Traditional approach to evaluation of candidate vaccines in clinical trials*

\begin{tabular}{|c|c|c|c|}
\hline $\begin{array}{l}\text { Phase of vaccine } \\
\text { clinical trial }\end{array}$ & Objectives of the trial & $\begin{array}{l}\text { Subject } \\
\text { number }\end{array}$ & $\begin{array}{l}\text { Typical } \\
\text { duration }\end{array}$ \\
\hline Phase I & Safety, tolerability, dose ranging and evidence of immunogenicity & $20-80$ & $1-2$ years \\
\hline Phase II & $\begin{array}{l}\text { Safety, dose optimization and immunogenicity as signals of potential } \\
\text { efficacy }\end{array}$ & $100-300$ & $2+$ years \\
\hline Phase Ilb & $\begin{array}{l}\text { Proof of concept trial (evidence of efficacy and safety); can be used } \\
\text { to determine which candidates advance to Phase III }\end{array}$ & $1,000-5,000+$ & $2+$ years \\
\hline Phase III & $\begin{array}{l}\text { Large placebo-controlled trial for efficacy and safety, with intention } \\
\text { to collect data for licensing }\end{array}$ & $2,000-5,000+$ & $4+$ years \\
\hline Phase IV & Post-licensing; further definition of risks, benefits and optimal use & Variable & Variable \\
\hline
\end{tabular}

Vaccines are licensed based on a risk-benefit analysis of the candidate vaccine safety profile, efficacy observed in Phase III clinical trials and public health need. In some cases, a known correlate of human protection defined for a particular pathogen by earlier Phase III trials can be used to predict efficacy and support licensing for subsequent vaccine formulations. Because of its sporadic nature, the incidence of Ebola virus infection in selected human populations is not predictable and does not allow for adequate statistical powering of a Phase III efficacy trial. Moreover, immune correlates of protection from disease in humans remain unknown and therefore cannot be used to assess candidate vaccine efficacy. To facilitate the licensing of drugs and biologics when efficacy cannot be evaluated in the setting of natural infection, the US Food and Drug Administration (FDA) promulgated a new regulation in 2002 as an alternative licensing pathway for pharmaceutical products that target highly lethal pathogens. The FDA's 'animal rule' allows for approval based on animal efficacy data conducted under Good Laboratory Practice controls, and human safety and immunogenicity data conducted under Good Clinical Practice controls, that support the correlate of protection defined in the animal model (described in the FDA Code of Federal Regulations CFR 21 314.600; see Further information). The animal rule is intended to be used as a pathway for regulatory review only when there is no other way to licence a vaccine ${ }^{3}$.

Immune correlate

A specific immune response to a vaccine that is closely associated with protection against infection, disease or some other defined end point.

Good Laboratory Practice A set of principles that provides a framework within which laboratory studies are planned, performed, monitored, recorded, reported and archived.

Good Clinical Practice

An ethical and scientific quality standard for designing,

conducting, recording and reporting trials that involves the participation of humans.

Application of the animal rule to a vaccine for Ebola virus is reasonable, and is probably the only way to develop a filovirus vaccine for human use, although the additional complexity of performing Good Laboratory Practice experiments under biosafety level 4 containment will be challenging. This approach requires an understanding of species used to predict human efficacy. The end point in an animal efficacy study must correlate with the desired effect in humans, which is typically a survival benefit or attenuation of severe disease, and allow determination of an effective dose-response relationship in animals and humans. FDA regulatory review and approval will require data substantiating that the vaccine under consideration is reasonably likely to provide a benefit in humans. In the case of therapeutic drugs, animal model data presented to substantiate a predicted therapeutic benefit must demonstrate effects at the biochemical level through elucidation of the mechanism of pathogen toxicity. For example, implementation of the animal rule to license pyridostigmine bromide in 2003 for the pretreatment of Soman nerve gas exposure was based on data from rhesus macaques because Soman pathophysiology is well characterized in this species ${ }^{3}$ (see Further information for a link to Drug Information from the Centre for Drug Evaluation and Research). In rhesus macaques, Soman irreversibly blocks acetylcholinesterase, an enzyme necessary for proper neurotransmission; data to support the licensing of pyridostigmine bromide provided evidence that it is a competitive and reversible blocker of Soman that binds its target enzyme.

Unlike pharmacological therapeutics, vaccines are not designed to block molecular targets, but instead, interrupt the pathogen replication cycle by preventing infection or clearing virus-infected cells through natural host defence mechanisms that constitute protective immunity. Licensing through the animal rule requires data showing that markers or correlates of protective vaccine-induced immunity are predictive for survival in an exposed host. Identification of immune correlates in animals has not been a requirement for FDA approval of human vaccines and, to date, no vaccines have been licensed under the animal rule. This paradigm therefore presents a challenge for basic, translational and clinical scientists to identify one or more parameters of primary immunity in animals that predict immunological protection against infection and can be tested in human clinical trials. pathophysiology and immune protection in the animal

\section{Immune correlates of vaccine protection}

Immune correlates of protection vary depending on the mode of vaccination, the route of infection and the nature of the immunological measurement. An immune assessment associated with disease outcome does not specify a mechanistic relationship between the immune marker and protection. Immunological correlates associated with efficacy vary in the strength of the quantitative and predictive relationship between a specific immune response and disease outcome $e^{4-6}$. For the purpose of this Review, we use the definition of a correlate provided in the scholarly review by Plotkin": a "specific immune response to a vaccine that is closely [associated with] 


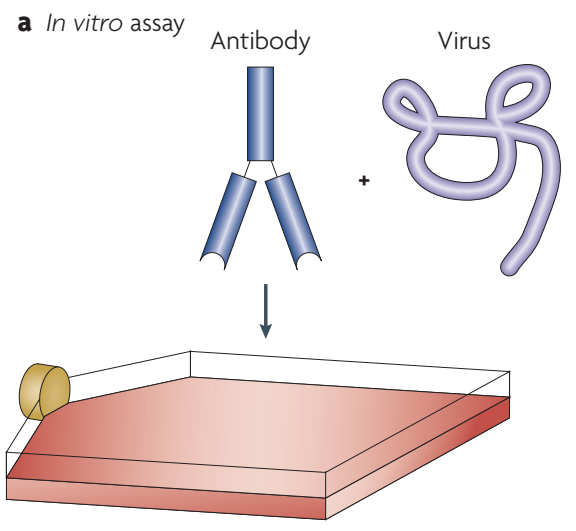

In vitro virus neutralization
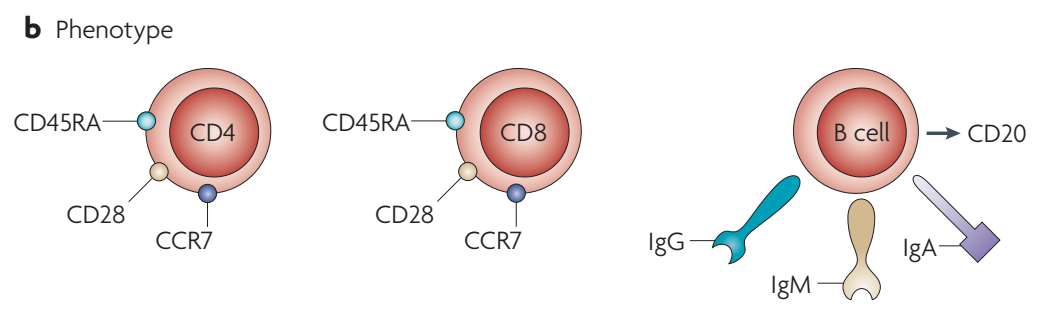

c Function
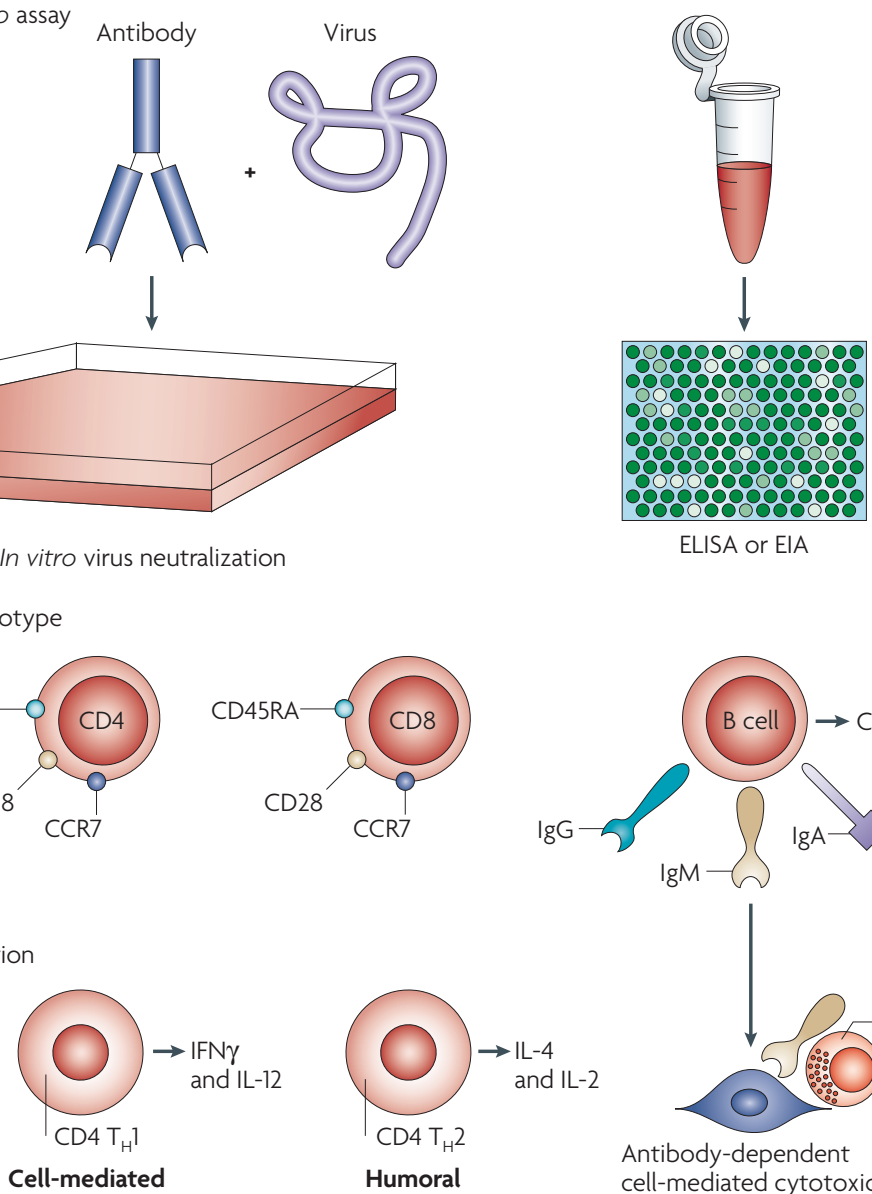

ELISA or EIA

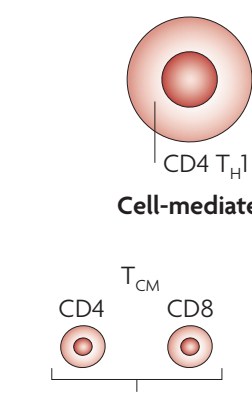

$\mathrm{TNF} \alpha+\mathrm{IFN} \gamma+\mathrm{IL}-2$

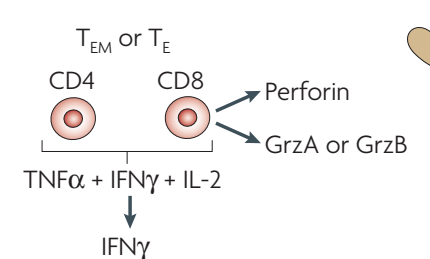
Antibody-dependent
cell-mediated cytotoxicity

Figure 1 | Determination of immunological end points that correlate with vaccine efficacy. Standard assays for the determination of immune markers include those that measure humoral immunity, such as in vitro virus neutralization, ELISA (enzyme-linked immunosorbent assay) and EIA (enzyme immunoassay) (a), and others that measure cell-mediated responses, including antigen-stimulated proliferation or cytolytic activity. Correlates can also be sought in additional phenotypic (b) and functional immune markers (c) that associate with vaccine efficacy. T cell lineage markers, cytokine secretion, antibody class and effector functions can individually, or in combination, segregate with host survival outcome. Grz, granzyme; IFN, interferon; Ig,

immunoglobulin; IL, interleukin; NK, natural killer; $\mathrm{T}_{C M}$, $T$ cell memory; $\mathrm{T}_{\mathrm{E}}$, $\mathrm{T}$ cell terminal effector; $\mathrm{T}_{\mathrm{EM}}, \mathrm{T}$ cell effector memory; $\mathrm{T}_{\mathrm{H}}, \mathrm{T}$ helper; $\mathrm{TNF}$, tumour necrosis factor.

protection against infection, disease, or other defined endpoint." Given the multifactorial nature of immunological protection, the most reliable correlate of immunity is also a determinant of protection, and reflects the underlying immune mechanisms responsible for resistance to infection. However, the correlate may be associated with protection without being causally related to virus clearance. The strength of the correlation required to support the licensing of an Ebola vaccine is

not specified in the animal rule, but is balanced against pathogen lethality, the risks of infection, the complexity of the immune responses that mediate protection and the urgency of the public health need.

Ebola virus outbreaks are unpredictable and survival is low, so it has not been possible to define human correlates of immunity to natural infection. Retrospective studies in the rare subjects who clear Ebola virus infection have identified qualitative associations between immune end points and survival ${ }^{7-16}$, but such studies have not identified predictive markers or surrogates of protective immunity. For the purposes of Ebola vaccine development, immune correlates of survival can only be established when the pathophysiology of an animal model closely resembles that of human disease. The selection of immunological end points for correlate assessment is informed by knowledge of the underlying mechanism of protection, a subject that remains under investigation for Ebola virus. The potential immune effectors involved in virus clearance may be dependent on one arm of the immune system (for example, cellular or humoral immunity). Alternatively, they may be dependent on various components of the innate and adaptive immune response. Determination of immune correlates of protection must therefore evaluate a range of phenotypic and functional markers of immunity (FIG. 1). T cell phenotype can be assessed with precision on a single-cell level by exploiting markers of lineage, homing profile and memory phenotype, such as $\underline{\text { CD } 4}$, $\underline{\text { CCR7 }}$ and CD28, respectively, among others. Such phenotypes can be defined further by their functional profiles (for example, those that relate to $\mathrm{T}$ helper function or surface markers associated with central T cell memory, T cell effector memory and $\mathrm{T}$ cell terminal effector phenotypes). Similarly, B cell immunological end points are captured using phenotypic markers, including CD20 expression and immunoglobulin (Ig) class, and functional assays that measure in vitro effector characteristics, such as direct virus neutralization or antibody-dependent cellular cytotoxicity.

Correlates of vaccine efficacy can be affected by variables such as the structure or stability of a specific immunogen, but can also be influenced by the mode of antigen delivery and processing in the host. Therefore, selection of immune end points is focused initially on mediators that are relevant to the vaccine platform. For example, protein formulations are often processed through endocytic pathways that stimulate $\mathrm{CD} 4^{+} \mathrm{T}$ helper $2\left(\mathrm{~T}_{\mathrm{H}} 2\right)$ cell responses and promote antibody production. By contrast, gene-based vaccines allow synthesis of foreign proteins within cells, which leads to processing of antigens through the proteasome, a process that more effectively elicits $\mathrm{CD}^{+} \mathrm{T}$ cell responses, while also eliciting antibody responses. Some gene-based vaccines have the potential to generate broad responses because of their ability to target antigen-presenting cells (APCs) directly, which is a property of certain viral vectors. The quality and range of vaccine-induced immune responses can therefore be influenced by the specificity of viral vectors for different APC targets, and must be determined empirically in animal models of vaccine protection for which immune reagents and assays are available. 
Table 2 | Immune correlates as a basis to optimize vaccine strategies

\begin{tabular}{llll} 
Vaccine & \multicolumn{3}{l}{ ELISA IgG titre* } \\
\hline Prime & Boost & Mouse & Macaque \\
\hline DNA & rAd & 200,000 & 75,000 \\
rAd & rAd & 100,000 & Not determined \\
\hline rAd & None & 16,000 & Not determined \\
\hline DNA & DNA & 12,000 & Not determined \\
\hline DNA & None & 6,400 & 2,000
\end{tabular}

*End point antibody titres were measured in sera from mice immunized with different combinations of DNA and recombinant adenovirus $(\mathrm{r} A \mathrm{~A})$ vectors encoding Ebola virus proteins, and used to prioritize candidate vaccines for testing in cynomolgus macaques. ELISA, enzyme-linked immunosorbent assay; Ig, immunoglobulin.

\section{Animal models of Ebola virus infection}

Protective immunity and immune correlates against Ebola virus infection have been best defined in mice, owing to the availability of inbred strains, which has allowed the quantitation of vaccine-induced humoral and cellular responses. A guinea pig infectious challenge model has also allowed us to define immune correlates in a rodent model that shares closely related coagulation defects with those observed in primates ${ }^{17}$. Xu and colleagues $^{18}$ used plasmid DNA vectors that encode sequences for the Ebola virus envelope glycoprotein, $\mathrm{GP}$, expressed on the surface of virions and virus-infected cells, and NP, the viral nucleocapsid protein that forms organized lattices when synthesized intracellularly ${ }^{19-21}$. Intramuscular vaccination with GP vectors generated potent $\mathrm{CD}^{+}$cytolytic responses 6 weeks after the first immunization in mice. Both GP and NP vectors induced high titre antigen-specific IgG antibodies that were present before infectious challenge and correlated with survival in guinea pigs. Although antibody levels provided a quantitative marker of immune protection, passive transfer of hyperimmune sera from these animals did not protect naive recipients. Similarly, a role for Ebola virus IgG as a correlate of vaccine protection was observed after mouse or guinea pig immunization with Venezuelan equine encephalitis GP and NP vaccines ${ }^{22,23}$. These results suggested that IgG did not completely block infection, required cofactors that were not present in the serum or lacked appropriate effector functions that were dependent on affinity or epitope specificity ${ }^{24}$. In support of the last possibility, other monoclonal antibodies or polyclonal Ig from survivors of active infection in either humans or mice showed success in protecting naive mice from disease and death ${ }^{25,26}$, confirming that Ebola virusdirected antibody quality is variable and is influenced by the way in which it was generated.

Evidence from rodent models of Ebola virus infection suggested that vaccine-induced IgG against Ebola virus GP is a quantitative immune end point that reflects the magnitude of underlying combined $B$ and $T$ cell vaccine responses. It is likely that cellular responses are present after immunization, but currently available methods for assessing these end points lack the sensitivity or specificity for reliable detection. Indeed, a necessary role for $\mathrm{CD}^{+}$and $\mathrm{CD}^{+} \mathrm{T}$ cell functions is unambiguous from genetic knockout and adoptive transfer studies in mice in which these components have been specifically targeted for deletion or enhancement, respectively ${ }^{27-29}$. Likewise, natural killer cells also seem to functionally complement vaccine-induced Ebola virus antibodies for virus clearance in mice $^{30}$.

\section{Immune correlates of vaccine protection}

The murine model of Ebola virus infection has revealed pathways and mechanisms of immune protection that are unparalleled in other animal models. However, vaccine approval for human use requires the use of a species that reliably reflects human disease, such as a non-human primate. A number of aspects of the Ebola virus-host interaction differ between murine and human infections; for example, to infect mice, virus isolates that infect humans must be adapted by serial passage. In this regard, Ebola virus infection in non-human primates more closely mimics disease pathogenesis observed in humans. Therefore, immune correlates of vaccine protection must be defined in non-human primates despite the relative paucity of reagents that are available for discerning and manipulating primate mediators of immune protection. Discordant results of vaccine efficacy studies between mice and non-human primates have been observed with Ebola vaccines. The importance of ELISA (enzyme-linked immunosorbent assay) IgG as an immunological end point established by DNA vaccination in mice guided investigators to augment genetic vaccine strategies with viral vectors that were capable of eliciting higher-magnitude antibody responses in primates than was possible with plasmid DNA alone. Alternative vector combinations were screened in mice to identify the regimen that yielded the highest ELISA IgG titres against Ebola virus GP (Table 2). Successful immune protection of non-human primates was accomplished using a heterologous prime-boost vaccine with DNA and replication-defective recombinant adenovirus (rAd) vectors encoding Ebola virus GP and NP ${ }^{31}$, the platform that shows the highest potency for eliciting antigen-specific antibodies. The first studies that used the DNA-rAd vaccine in non-human primates efficiently induced high-titre antibodies and CD4 ${ }^{+}$ T cells before infectious challenge. Subsequent assessment of a single-shot rAd Ebola vaccine (omitting the DNA priming step) confirmed the presence of antigenspecific ELISA IgG in survivors and demonstrated the reproducibility of the antibody response as a correlate of protection. Furthermore, other Ebola vaccines developed for macaques based on vesicular stomatitis virus ${ }^{32}$, parainfluenza virus ${ }^{33}$ or virus-like particles (VLPs) ${ }^{34}$ have since reported that protective immunity correlated with the presence of virus-specific antibodies in all vaccinated survivors of infectious challenge. The efficacy of these additional vaccines in non-human primates clearly establishes that protective immunity to Ebola virus infection can be achieved and offers the opportunity for further detection of immune correlates. Whether immune correlates of protection will apply across vaccine platforms remains unknown and may depend on whether these different vaccine vectors elicit related pathways of immunity to confer protection against challenge, but it is 
Table 3 | Vaccines inducing uniform protective immunity in non-human primates

\begin{tabular}{|c|c|c|c|c|c|}
\hline \multirow[t]{2}{*}{ Approach } & \multirow[t]{2}{*}{ Virus* } & \multirow[t]{2}{*}{ Antigen $^{\neq}$} & \multicolumn{2}{|c|}{ Immunity ${ }^{\S}$} & \multirow[t]{2}{*}{ Refs } \\
\hline & & & Humoral & Cellular & \\
\hline \multicolumn{6}{|c|}{ Whole virus or subunit } \\
\hline Virus-like particle & Ebola virus & $\mathrm{GP}+\mathrm{VP} 40+\mathrm{NP}$ & $\lg G$ & (CD4 or CD8) & 34 \\
\hline \multicolumn{6}{|l|}{ Gene-based } \\
\hline Adenovirus & Ebola virus and Marburg virus & GP & $\lg G$ & (CD8) & 44,45 \\
\hline DNA & Marburg virus & GP & $\lg G$ & CD4 and (CD8) & $\begin{array}{r}\text { N.J.S., } \\
\text { unpublished } \\
\text { observations }\end{array}$ \\
\hline DNA or adenovirus & Ebola virus and Marburg virus & $\mathrm{GP}+\mathrm{NP}$ & $\lg G$ & CD4 & 31 \\
\hline Parainfluenza & Ebola virus & $\mathrm{GP}$ and $\mathrm{GP}+\mathrm{NP}$ & $\lg A$ & (CD4) & 33 \\
\hline $\begin{array}{l}\text { Venezuelan equine } \\
\text { encephalitis virus }\end{array}$ & Marburg virus & $\mathrm{GP}$ and $\mathrm{GP}+\mathrm{NP}$ & $\lg G$ & Unknown & 43 \\
\hline $\begin{array}{l}\text { Vesicular stomatitis } \\
\text { virus }\end{array}$ & Ebola virus and Marburg virus & GP & $\lg G$ & Unknown & 32 \\
\hline \multicolumn{6}{|c|}{$\begin{array}{l}\text { *Immunization strategies that have yielded } 100 \% \text { protection in non-human primates are shown along with the challenge virus, } \\
\text { Ebola virus or Marburg virus. } ₹ \text { Vaccines contained protein or coding sequences for the different virus antigens; glycoprotein (GP) } \\
\text { was included in all successful strategies. \$Pre-challenge cellular immune responses were observed sporadically (parentheses } \\
\text { indicate partial responses), but ELISA (enzyme-linked immunosorbent assay) immunoglobulin G (lgG) was a consistent indicator of } \\
\text { vaccine-induced protective immunity. NP, nucleoprotein, }\end{array}$} \\
\hline
\end{tabular}

noteworthy that each of the vaccines shown to be $100 \%$ effective for protection of non-human primates induced antibody responses (TABLE 3).

The efficacy of non-human primate vaccines has been most consistently associated with the presence of ELISA IgG before infectious challenge. Because the pathophysiology and clinical symptoms of non-human primate Ebola virus infection accurately recapitulates human Ebola virus infection ${ }^{35}$, this observation provides an important lead in the development of Ebola vaccines for human use. This animal model therefore satisfies the requirements for vaccine licensing using the animal rule. However, as stated at the beginning of this Review, vaccine regulatory review will be facilitated by quantitative measurements of immunity that predict protection from infection. As a rigorous test for the strength of ELISA IgG as a correlate of immune protection, the predictive value of IgG titres was evaluated using sera from non-human primate subjects immunized with rAd5-based Ebola vaccines that encoded various combinations of GP and NP, as well as different mutated versions of GP. Nearly complete survival can be predicted in subjects that generate a particular pre-challenge titre of ELISA IgG against GP (FIG. 2). Most licensed vaccines have not demonstrated $100 \%$ protective efficacy before FDA approval, so identification of a precise Ebola virus IgG threshold titre will be dependent on the level of vaccine efficacy specified. A survival rate of $85 \%$ (FIG. 3) establishes a lower threshold for the immune correlate of protection (FIG. 2). Requirements for vaccine efficacy will probably be balanced against risks for mortality in different exposure situations.

ELISA IgG serves as a useful example of an immune correlate of protection in cynomolgus macaques after immunization with rAd5 vaccines. However, it may not be the definitive correlate that bridges the gap between animal and human immune responses for vaccine licensing. The question remains of whether additional correlates can increase the value of ELISA IgG to predict protective immunity against Ebola virus. Those subjects that mount quantitatively similar antibody titres but exhibit different survival outcomes offer an opportunity to explore functional attributes of antibodies that may enhance the distinction between survivors and fatalities based on antibody quality. Although direct in vitro virus neutralization assays are not predictive for protection in non-human primates, this effector function has occasionally been reported in samples from Ebola vaccine-immunized macaques ${ }^{32}$. More recently, antibody-dependent cellular cytotoxicity was detected after vaccination with $\mathrm{VLPs}^{34}$. Neither of these effector functions was a quantitative correlate of protective immunity. However, the data imply that ELISA IgG titres may indeed reflect the activity of underlying immunological components.

In addition to the role for $\mathrm{T}$ cells in antibody effector function, the mechanism by which the immune system confers protection is also likely to involve T cells. Although ELISA IgG is a quantitative and reliable correlate for vaccine efficacy, and immune protection can be transferred from immune serum to naive rodents, passive transfer failed to protect non-human primates, suggesting that the mechanism of immune protection against Ebola virus infection in primates is more complex than in rodents ${ }^{26,36}$ (N.J.S., unpublished observations). Furthermore, mouse adoptive transfer and knockout studies that implicated both $\mathrm{B}$ and $\mathrm{T}$ cell mediators of protective immunity suggest that the mechanism of vaccine-generated Ebola virus immunity is probably multifaceted, even in mice. The mechanism of protection in primates might be similarly complex. Indeed, preliminary immunodepletion studies of T cell subsets in macaques suggest that cellular immunity is 


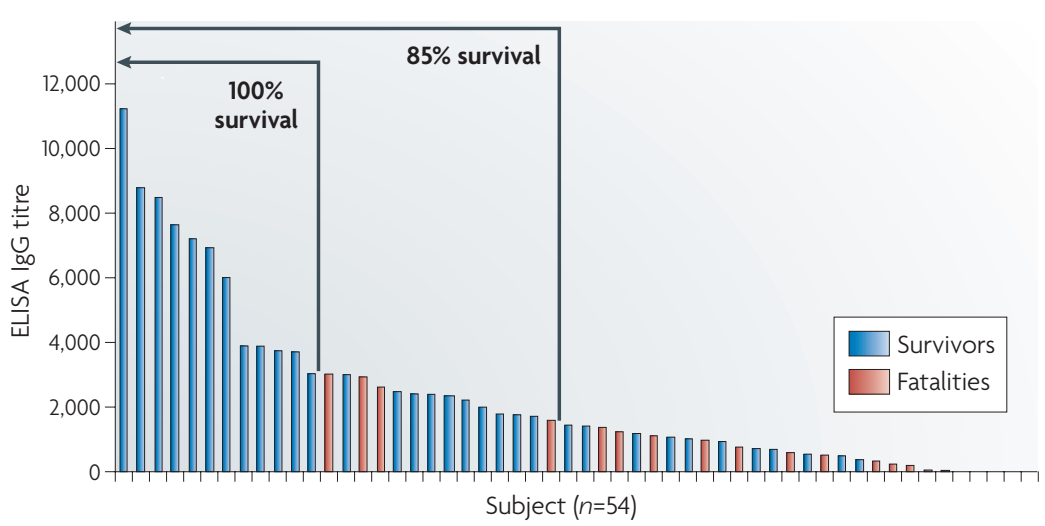

Figure 2 | Assessment of antigen-specific immunoglobulin $\mathrm{G}(\mathrm{IgG})$ as a correlate of Ebola vaccine efficacy. Immune sera from macaques immunized with genetic vaccines encoding wild-type or mutant Ebola virus glycoprotein alone or in combination with nucleoprotein showed a spectrum of $\mathrm{lg} \mathrm{G}$ antibody titres measured by ELISA (enzyme-linked immunosorbent assay). Antibody titre was a quantitative predictor (surrogate) for vaccine efficacy; $100 \%$ immune protection was predicted by an antibody titre of 1:3,700. analyses, they may prove to be beneficial complements to ELISA IgG for prediction of vaccine efficacy. To achieve a determinant of immunity for filoviruses, we may need to measure a combination of specific immunological functions that include both antibody and cellular responses. For filoviruses and other viruses, such as HIV-1, herpes simplex virus and hepatitis C virus, this may require discovery of new approaches for measuring cellular immunity, and as the complexity of protective immunity increases it is less likely that the measurements will translate accurately from animal models to humans. Therefore, the immediate goal in filovirus vaccine development is to define a reliable correlate that can be used to support licensing of a vaccine based on the animal rule. Ultimately, the strength of an antibody or $\mathrm{T}$ cell correlate will have to be confirmed in human clinical trials, using the final candidate vaccine in a comparative analysis of non-human primate and human immune responses.

Immune correlates of Ebola vaccine immunity in non-human primates provide a basis for evaluating a vaccine that protects against lethal challenge for its immunogenicity in humans. Although further confirmation could theoretically be derived from survivors of natural Ebola virus infection, such subjects are rare and the time from recovery is variable. After the first outbreak of Ebola virus, the total survivor cohort constituted only $12 \%$ of identified cases. Therefore, analyses of human immune responses have been performed only retrospectively, at variable times and in small numbers of subjects. It is noteworthy that the presence of Ebola virus-specific ELISA IgG has been associated with survival from natural infection? ${ }^{7}$. Additional immunological markers that segregate with human survival from natural infection include specific human leukocyte antigen, class B alleles ${ }^{15}$ and $\mathrm{T}$ cell activation as assessed by cytokine production $^{12,13}$, but these correlative parameters are observational, and therefore fall short of defining predictive indicators of protective immunity. In addition, it is not possible to discern whether the surveyed immunological end points reflect mediators of protection or are simply memory responses generated by exposure to virus.

Ultimately, the licensing of vaccines for human use will require bridging of the immune correlate between pivotal animal efficacy studies and placebocontrolled clinical trials in human volunteers (FIG. 3). To this end, a Phase I randomized human clinical trial has been completed for an Ebola DNA vaccine. The first Ebola vaccine clinical trial showed that all 20 of the vaccinated individuals who were immunized with three doses generated positive antibody titres as measured by ELISA against GP or NP, and antibody was present in all individuals regardless of vaccine dose, which ranged from 2 to $8 \mathrm{mg}$ of $\mathrm{DNA}^{40}$. The range of antibody titres was similar to those observed in nonhuman primates that were vaccinated with a similar regimen $^{31}$, indicating that the monkey model is a valid predictor of human immune responses against Ebola virus antigens. 


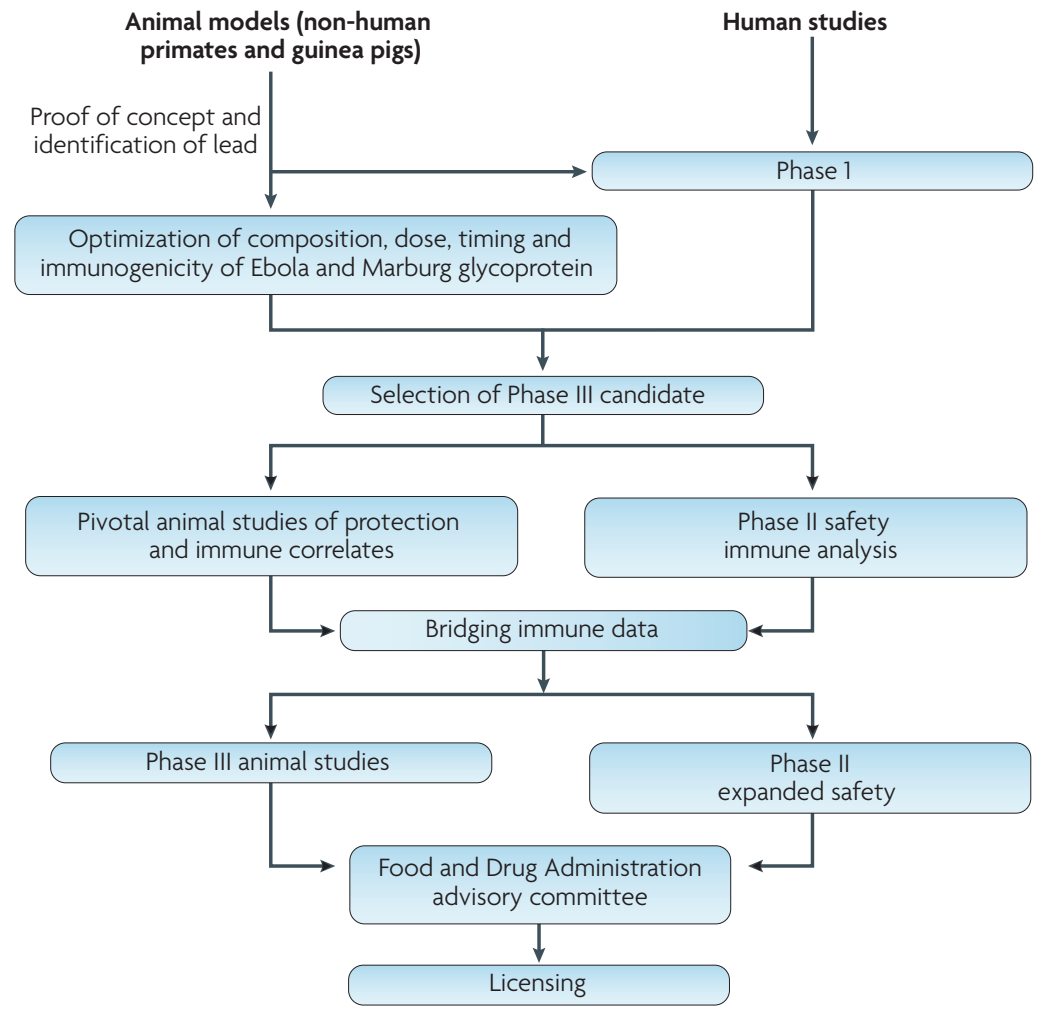

Figure 3 | Ebola vaccine development pathway using the animal rule. Early vaccine candidates are optimized for immune potency in small animal models. Selected candidates are evaluated further in an animal model that adequately represents infection in humans (cynomolgus macaques) to establish an immune correlate of vaccine efficacy, and a pivotal animal study is performed to bridge the immune correlate between macaque efficacy studies and human Phase II clinical trials. Data from Phase III animal studies and expanded Phase II human studies are compiled for regulatory review and vaccine licensing.
Application of the animal rule to other diseases Vaccine licensing using the animal rule will be applicable to a number of pathogens for which traditional clinical efficacy trials are not possible. Such diseases include those caused by highly pathogenic agents that are precluded from human testing because direct challenge would require exposing healthy human volunteers to a lethal substance or because populations that are at risk for natural infection cannot be identified for efficacy trials. The Centers for Disease Control proposed a list of category A priority pathogens, which would include viral agents, such as Ebola virus, Marburg virus and smallpox viruses, as well as bacterial agents, such as those that cause plague, botulism and anthrax. Although some of these agents do not pose an immediate public health concern in the United States, the animal rule is intended to provide a pathway for licensing of vaccines for use worldwide and against highly transmissible agents that could be used as bioweapons. In this way, vaccines or therapeutic interventions could be licensed in advance of a threat and stockpiled for use in an emergency. In addition, the rule would also prove useful for natural infections that are episodic and unpredictable in nature, such as West Nile virus, avian influenza or SARS (severe acute respiratory syndrome). Such diseases threaten public health episodically, but have not yet become established in a sufficiently large population to allow clinical efficacy studies.

\section{Concluding remarks}

To date, no vaccines have received regulatory approval and been licensed using the FDA animal rule. Here, we have provided a framework for understanding this regulatory pathway and how studies of vaccine-induced protective immune responses in animals might provide support for licensing. This pathway does not diminish the level of regulatory review required for vaccine approval; extensive human testing is still required to demonstrate safety and immunogenicity. Furthermore, the data in support of licensing may actually exceed the requirements of the traditional approval process, as an immune correlate of vaccine protection must also be revealed, which is not required for currently used licensed US vaccines. The predictive relationship between animals and humans for protective efficacy is unknown, and therefore an immune correlate is used to bridge the gap between animal efficacy studies and human immunogenicity trials. It has not yet been determined what level of efficacy in animals will be required for vaccine approval, but vaccines currently administered to the US population have provided efficacies in human trials that are as low as $18 \%$. Higher potency may be required for vaccines approved using the animal rule because of the expectations that they would be highly effective and useful in an emergency, but even this level of efficacy will provide a substantial benefit against pathogens with high mortality and therefore may be an acceptable level for countermeasures against emerging natural infections or deliberate biodefence threats. 
1. Ascenzi, P. et al. Ebolavirus and Marburgvirus: insight the Filoviridae family. Mol. Aspects Med. 29 , 151-185 (2008)

2. Towner, J. in 4th Int. Symp. Ebola and Marburg Viruses, CIRMF Global Symposium (Libreville, Gabon, 2008)

3. Crawford, L. M. New drug and biological drug products; evidence needed to demonstrate effectiveness of new drugs when human efficacy studies are not ethical or feasible. Fed. Regist. 67, 37988-37998 (2002)

4. Plotkin, S. A. Vaccines: correlates of vaccine-induced immunity. Clin. Infect. Dis. 47, 401-409 (2008). Plotkin provides a concise synthesis of historical data regarding vaccine-induced correlates of protection and proposes a useful nomenclature for defining such associations.

5. Oin, L., Gilbert, P. B., Corey, L., McElrath, M. J. \& Self, S. G. A framework for assessing immunological correlates of protection in vaccine trials. J. Infect. Dis. 196, 1304-1312 (2007).

6. Sadoff, J. C. \& Wittes, J. Correlates, surrogates, and vaccines. J. Infect. Dis. 196, 1279-1281 (2007)

7. Baize, S. et al. Defective humoral responses and extensive intravascular apoptosis are associated with fatal outcome in Ebola virus-infected patients. Nature Med. 5, 423-426 (1999).

This study was one of the first to demonstrate an association between immunological markers and disease outcome for Ebola virus infection.

8. Baize, S. et al. Inflammatory responses in Ebola virusinfected patients. Clin. Exp. Immunol. 128, 163-168 (2002).

9. Busico, K. M. et al. Prevalence of IgG antibodies to Ebola virus in individuals during an Ebola outbreak, Democratic Republic of the Congo, 1995. J. Infect. Dis. 179 (Suppl. 1), 102-107 (1999)

10. Hutchinson, K. L. \& Rollin, P. E. Cytokine and chemokine expression in humans infected with Sudan Ebola virus. J. Infect. Dis. 196 (Suppl. 2), 357-363 (2007).

11. Ksiazek, T. G. et al. Clinical virology of Ebola hemorrhagic fever (EHF): virus, virus antigen, and IgC and IgM antibody findings among EHF patients in Kikwit, Democratic Republic of the Congo, 1995. J. Infect. Dis. 179 (Suppl. 1), 177-187 (1999).

12. Leroy, E. M. et al. Human asymptomatic Ebola infection and strong inflammatory response. Lancet 355, 2210-2215 (2000)

13 Leroy, E. M., Baize, S., Debre, P., Lansoud-Soukate, J. ¿ Mavoungou, E. Early immune responses accompanying human asymptomatic Ebola infections. Clin. Exp. Immunol. 124, 453-460 (2001).

14. Rowe, A. K. et al. Clinical, virologic, and immunologic follow-up of convalescent Ebola hemorrhagic fever patients and their household contacts, Kikwit, Democratic Republic of the Congo. Commission de Lutte contre les Epidemies a Kikwit. J. Infect. Dis. 179 (Suppl. 1), 28-35 (1999).

15. Sanchez, A., Wagoner, K. E. \& Rollin, P. E. Sequencebased human leukocyte antigen-B typing of patients infected with Ebola virus in Uganda in 2000 : identification of alleles associated with fatal and nonfatal disease outcomes. J. Infect. Dis. 196 (Suppl. 2), 329-336 (2007)

16. Villinger, F. et al. Markedly elevated levels of interferon (IFN)- $\gamma$, IFN- $\alpha$, interleukin (IL)-2, IL-10, and tumor necrosis factor- $\alpha$ associated with fatal Ebola virus infection. J. Infect. Dis. 179 S188-S191 (1999). This paper describes soluble markers that segregate with survival outcome after Ebola virus infection.
17. Bente, D., Gren, J., Strong, J. E. \& Feldmann, H. Disease modeling for Ebola and Marburg viruses. Dis. Model. Mech. 2, 12-17 (2009).

18. Xu, L. et al. Immunization for Ebola virus infection Nature Med. 4, 37-42 (1998).

First demonstration of vaccine-induced protective immunity against Ebola virus.

19. Huang, Y., Xu, L., Sun, Y. \& Nabel, G. J. The assembly of Ebola virus nucleocapsid requires virion-associated proteins 35 and 24 and posttranslational modification of nucleoprotein. Mol. Cell 10, 307-316 (2002).

20. Johnson, R. F., Bell, P. \& Harty, R. N. Effect of Ebola virus proteins GP, NP and VP35 on VP40 VLP morphology. Virol. J. 3, 31 (2006).

21. Licata, J. M., Johnson, R. F., Han, Z. \& Harty, R. N. Contribution of Ebola virus glycoprotein nucleoprotein, and VP24 to budding of VP40 virus-like particles. J. Virol. 78, 7344-7351 (2004).

22. Pushko, P. et al. Recombinant RNA replicons derived from attenuated Venezuelan equine encephalitis virus protect guinea pigs and mice from Ebola hemorrhagic fever virus. Vaccine 19, 142-153 (2000)

23. Wilson, J. A. \& Hart, M. K. Protection from Ebola virus mediated by cytotoxic T lymphocytes specific for the viral nucleoprotein. J. Virol. 75, 2660-2664 (2001). This study showed that CTLs mediate immune protection against Ebola virus infection.

24. Wilson, J. A. et al. Epitopes involved in antibody mediated protection from Ebola virus. Science $\mathbf{2 8 7}$ 1664-1666 (2000).

This study showed that antigen-specific antibodies could provide protection from Ebola virus infection.

25. Gupta, M., Mahanty, S., Bray, M., Ahmed, R. \& Rollin, P. E. Passive transfer of antibodies protects immunocompetent and immunodeficient mice against lethal Ebola virus infection without complete inhibition of viral replication. J. Virol. 75, 4649-4654 (2001).

26. Oswald, W. B. et al. Neutralizing antibody fails to impact the course of Ebola virus infection in monkeys. PLoS Pathog. 3, e9 (2007)

27. Gupta, M. et al. Persistent infection with Ebola virus under conditions of partial immunity. J. Virol. 78, 958-967 (2004).

28. Gupta, M. et al. CD8-mediated protection against Ebola virus infection is perforin dependent. J. Immunol. 174, 4198-4202 (2005).

29. Warfield, K. L. et al. Induction of humoral and CD8+ $\mathrm{T}$ cell responses are required for protection against lethal Ebola virus infection. J. Immunol. 175 1184-1191 (2005).

30. Warfield, K. L. et al. Role of natural killer cells in innate protection against lethal Ebola virus infection J. Exp. Med. 200, 169-179 (2004).

31. Sullivan, N. J., Sanchez, A., Rollin, P. E., Yang, Z.-Y. \& Nabel, G. J. Development of a preventive vaccine for Ebola virus infection in primates. Nature 408 605-609 (2000)

32. Jones, S. M. et al. Live attenuated recombinant vaccine protects nonhuman primates against Ebola and Marburg viruses. Nature Med. 11, 786-790 (2005)

33. Bukreyev, A. et al. Successful topical respiratory tract immunization of primates against Ebola virus. J. Virol. 81, 6379-6388 (2007)

34. Warfield, K. L. et al. Ebola virus-like particle-based vaccine protects nonhuman primates against lethal Ebola virus challenge. J. Infect. Dis. 196 (Suppl. 2), 430-437 (2007)

35. Geisbert, T. W. et al. Pathogenesis of Ebola hemorrhagic fever in cynomolgus macaques: evidence that dendritic cells are early and sustained targets of infection. Am. J. Pathol. 163, 2347-2370 (2003).
36. Jahrling, P. B. et al. Passive immunization of Ebola virus-infected cynomolgus monkeys with immunoglobulin from hyperimmune horses. Arch. Virol. Suppl. 11, 135-140 (1996).

37. Betts, M. R. et al. Sensitive and viable identification of antigen-specific $\mathrm{CD} 8{ }^{+} \mathrm{T}$ cells by a flow cytometric assay for degranulation. J. Immunol. Methods $\mathbf{2 8 1}$, 65-78 (2003)

Description of functional differences in $\mathrm{T}$ cell populations by polychromatic flow cytometry of cytokine responses.

38. Betts, M. R. et al. Characterization of functional and phenotypic changes in anti-Gag vaccine-induced T cell responses and their role in protection after HIV- 1 infection. Proc. Natl Acad. Sci. USA 102, 4512-4517 (2005).

39. Pitcher, C. J. et al. Development and homeostasis of T cell memory in rhesus macaque. J. Immunol. 168 29-43 (2002).

40. Martin, J. E et al. A DNA vaccine for Ebola virus is safe and immunogenic in a phase I clinical trial. Clin. Vaccine Immunol. 13, 1267-1277 (2006). First human clinical trial for an Ebola vaccine.

41. Edghill-Smith, Y. et al. Smallpox vaccine-induced antibodies are necessary and sufficient for protection against monkeypox virus. Nature Med. 11, 740-747 (2005).

42. Schmitz, J. E. et al. Control of viremia in simian immunodeficiency virus infection by CD8 lymphocytes. Science 283, 857-860 (1999)

43. Hevey, M., Negley, D., Pushko, P., Smith, J. \& Schmaljohn, A. Marburg virus vaccines based upon alphavirus replicons protect guinea pigs and nonhuman primates. Virology 251, 28-37 (1998).

44 Sullivan, N. J. et al. Accelerated vaccination for Ebola virus haemorrhagic fever in non-human primates. Nature 424, 681-684 (2003)

45. Swenson, D. L. et al. Vaccine to confer to nonhuman primates complete protection against multistrain Ebola and Marburg virus infections. Clin. Vaccine Immunol. 15, 460-467 (2008).

\section{Acknowledgements}

The authors thank $\mathrm{C}$. Asiedu for careful reading of the manuscript, A. Tislerics and D. Jeffers for help with manuscrip preparation and $\mathrm{M}$. Cichanowski and B. Hartman for assistance with the figures.

Competing interests statement

The authors declare competing financial interests: see web version for details.

DATABASES

UniProtKB: http://www.uniprot.or

CCR7 $|\underline{C D 20}| \underline{C D 4}|\underline{C D 28}| \underline{G P} \mid \underline{N P}$

FURTHER INFORMATION

Nancy J. Sullivan's homepage: http://www3.niaid.nih.gov/ labs/aboutlabs/VRC/biodefenseResearchLaboratory/ ClinicalTrials.gov: http://clinicaltrials.gov/ Drug Information from the Centre for Drug Evaluation and Research: http://www.fda.gov/cder/drug/infopage/ Pyridostigmine Bromide/default.htm

FDA Code of Federal Regulations CFR 21 314.600: http:// www.accessdata.fda.gov/SCRIPTs/cdrh/cfdocs/cfcfr/ CFRSearch.cfm?CFRPart=314\&showFR=1EsubpartNod $\mathrm{e}=21: 5 \cdot 0 \cdot 1 \cdot 1 \cdot 4 \cdot 9$

National Institute of Allergy and Infectious Diseases (Understanding Vaccine Clinical Studies): http://www3. niaid.nih.gov/Volunteer/VRC/understanding.htm ALL LINKS ARE ACTIVE IN THE ONLINE PDF 
CORRIGENDUM

Correlates of protective immunity for Ebola vaccines: implications for regulatory approval by the animal rule

Julie E. Martin, Barney S. Graham, Gary J. Nabel and Nancy J. Sullivan

Nature Reviews Microbiology 7, 393-400 (2009)

In the above article, the authors failed to acknowledge the technical assistance and data analysis of M. Bailey in the acknowledgements section. The authors apologize for this error. 